\title{
EDITORIAL
}

\section{Accepted or rejected?}

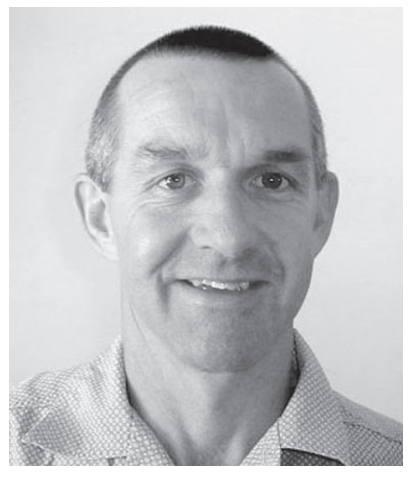

Despite the proliferation of new specialist journals it is becoming increasingly difficult to get research work published. There are a number of reasons for this - perhaps the most obvious is that there are more institutions which specialise in sports medicine and exercise science now than there were 10 years ago. Therefore the volume of research has increased precipitously. This means the standard of the journals can be kept at a reasonably high level because there is always a surplus of papers which are submitted for consideration.

Just as the other journals are raising the bar for the quality of the papers they publish, so is the South African Journal of Sports Medicine doing likewise. This has enabled us to refine our niche area and focus on those papers which are relevant to sports medicine and exercise science in South Africa. Coupled to this we have also become stricter about publishing research which attempts to determine 'ethnic differences' without controlling or accounting for factors such as nutritional status, level of physical activity and socio-economic status between the groups. Failing to do this makes the conclusions of any differences in ethnicity irrelevant and incorrect. Researchers who use ethnicity as a variable are encouraged to read the excellent article 'Racial profiling in medical research: what are we measuring?'. '

The South African Journal of Sports Medicine rejects about $50 \%$ of the papers submitted because they are either not formatted correctly or do not have a clearly outlined question. Another reason for rejecting papers is that the discussion on the data does not devote sufficient attention to the actual data in the study. In an attempt to assist in increasing the 'hit rate' of papers getting accepted I thought I would highlight a few simple points which are linked to the ultimate success of the paper getting published. Firstly the introduction - this is probably the most difficult section to write as this is the section of the paper that captures the interest of the reader. A well-written introduction should provide sufficient background about why the study was done, and then should explain very clearly the research question. If the introduction is lacking in any of these features, it makes it difficult for the rest of the paper to prove to the reader that the study is worthwhile. Next the methods have to be described in sufficient detail so that, if needs be, someone else can repeat the study with the same degree of accuracy. The authors need to find a balance between citing previously published methods and using an original description of the methodology.

The next important section is the results section. The creative ability of the researchers comes to the fore in this section as the data need to be displayed clearly and accurately. The authors have to find a balance between presenting the data in the text, figures and tables. Figures should be clearly drawn with clearly labelled axes and legends with sufficient detail to enable the figure and legend to 'stand alone'. Several authors make the mistake of discussing their findings in the results section. This is not acceptable and any paper with any discussion in the results section will be sent back for reformatting. The statistics used to analyse the data must be presented clearly. If the author(s) is/are not proficient in statistics then they are encouraged to consult a statistician who can assist in ensuring that the statistics are relevant and applied correctly. In many types of research in exercise science and sports medicine the researchers need to go beyond just presenting their data as being significant, or not, at the $5 \%$ level. Considering elite performance, in many cases an improvement of $0.5 \%$ is regarded as meaningful, yet it will be almost impossible to ever show that this is statistically significant. There are other creative procedures, such as effects sizes and magnitude of differences which allow the data to be interpreted in a more meaningful way. The last section of the paper is the discussion. A good discussion finds a balance between unfolding and interpreting the main findings in the study and relating these findings to other published research, where possible. A good discussion also ensures that the original research question, as outlined in the introduction, was answered effectively.

In 2009 the South African Journal of Sports Medicine will be adopting revised guidelines for authors. These will be published on the website (www.sajsm.org.za./index.php/sajsm). In accordance with the new guidelines, the editorial staff is going to be strict about the number of references with each paper. The reason for restricting the number of references is not to curtail information linked to the study, but rather it is a pragmatic decision which relates to the number of pages per edition and cost of publishing the Journal. Readers of the journal will rightly be unimpressed if a large proportion of the journal is dedicated to references, at the expense of new content.

All the people who reviewed papers in 2008 are thanked most sincerely - without exception the standard of the reviews was very high. Anyone who has reviewed for a journal knows that it is a thankless job - there is no payment or recognition, and it is just one of those commitments that one is obliged to make as a scientist. Having been on the receiving end many times myself, it is never pleasant to have a paper rejected. However, it makes it a bit easier to accept the decision if the standard of the review is high. So to all the reviewers, many thanks, your contribution to the South African Journal of Sports Medicine is greatly appreciated!

\author{
Mike Lambert \\ Editor-in-Chief \\ 1. Ncayiyana DJ. Racial profiling in medical research: what are we measuring? S Afr Med \\ J 2007; 97: 1225-1226.
}

